\title{
Discovering Chemical Site Occupancy- Modulus Correlations in Ni based Intermetallics via Statistical Learning Methods
}

\author{
Scott R. Broderick ${ }^{1}$, Aakash Kumar ${ }^{2,3, \#}$, Adedapo A. Oni ${ }^{4}$, James M. LeBeau ${ }^{4}$, Susan B. \\ Sinnott ${ }^{2,3}$, Krishna Rajan ${ }^{1, *}$ \\ ${ }^{1}$ Department of Materials Design and Innovation, University at Buffalo - The State University of New \\ York, Buffalo, NY 14260, USA \\ ${ }^{2}$ Department of Materials Science and Engineering, University of Florida, Gainesville, FL 32611, USA \\ ${ }^{3}$ Department of Materials Science and Engineering, The Pennsylvania State University, University Park, \\ PA 16802, USA \\ ${ }^{4}$ Department of Materials Science and Engineering, North Carolina State University, Raleigh, NC 37695, \\ USA \\ *Corresponding author: krajan3@buffalo.edu \\ \# Present Address: Department of Materials Science and Engineering, University of Pennsylvania, \\ Philadelphia, PA 19104, USA
}

\begin{abstract}
We show how one may extract spectral features from the density of states (DOS) of $\mathrm{L}_{2}-\mathrm{Ni}_{3} \mathrm{Al}$ alloys that can serve as signatures or electronic "fingerprints" which capture the correlation between site occupancy of dopants and elastic properties. Based on this correlation, we have developed a computational approach for rapidly identifying the impact of the selection of dopant chemistries on bulk moduli of intermetallics. Our results show for example that Cr preferentially occupies the $\mathrm{Al}$ site in $\mathrm{Ni}_{3} \mathrm{Al}$ which is confirmed by scanning transmission electron microscopy (STEM) energy dispersed X-ray spectroscopy (EDS) analysis. We further show that this preference is due to a sensitivity of $\mathrm{Cr}$ to the DOS at -1.7 and $0.2 \mathrm{eV}$ relative to the Fermi energy. In terms of similarity in chemistry-property correlations, we find $\mathrm{Cr}$ has a similar effect to Ce when occupying an Al site, while Cr occupying a Ni site has similar correlation as $\mathrm{La}$ on a $\mathrm{Ni}$ site. This logic can be utilized in targeted design of new alloy chemistries based on similar property correlations and for targeted DOS modification.
\end{abstract}

Keywords: Materials informatics, Density functional theory, Density of state, Ni-base alloys, Scanning transmission electron microscopy

\section{Introduction}

In this paper, we extract the signatures of the DOS spectra which dictate the elastic moduli of alloys. Further, the DOS signatures of alloying site preference are identified, with the focus here on Ni-based alloys. In our previous work, we developed the relationship between the chemistry and crystal structure and the DOS signatures based on the characteristics of the elemental constituents [1-3]. Combining these threads, we are able to accelerate the chemical design of multicomponent systems for targeted properties by developing chemistry-electronic structureproperty relationships, thereby reducing the number of electronic structure calculations required by rapidly screening the enormous chemical search space. 
Nickel-based superalloys, which are of interest for their high-temperature behavior, have two phases present: $\mathrm{Y}-\mathrm{Ni}$ matrix and $\mathrm{Y}^{\prime}-\mathrm{Ni}_{3} \mathrm{Al}$. The $\mathrm{Ni}_{3} \mathrm{Al}$ phase has an $\mathrm{L}_{2}$ structure with $\mathrm{Al}$ at the corners and $\mathrm{Ni}$ at the face centers, and is the principal reason for the high temperature strength of superalloys due to the yield stress anomaly, where the yield stress increases with increasing temperature up to a limit [4]. Different alloying elements can modify the elastic properties of $\mathrm{Ni}_{3} \mathrm{Al}$ [5-7], with the superalloys having a complex composition with generally around 10 alloying elements. It is important to investigate the alloying behavior of the different elements in terms of their site-preference and elastic properties. A method for predicting site preference of a dopant atom in this $\mathrm{Ni}_{3} \mathrm{Al}$ phase was suggested by Ruban and Skriver [8], based on the energy change of moving an atom $X$ from a Ni site to an Al site $(E X N i \rightarrow A l)$. Later, Gleeson et al. [9] showed that this single parameter $E X N i \rightarrow A l$ can be used to predict the site preference of a dopant atom. This parameter has been studied for many transition elements [7-10]. This method assumes the Wagner-Schottky model to be true [11] according to which the formation enthalpy of the ternary alloy $\mathrm{Ni}-\mathrm{Al}-\mathrm{X}$ is a linear function of the formation enthalpy of $\mathrm{Ni}_{3} \mathrm{Al}$ and that of the point defect.

$\Delta H=\Delta H N i 3 A l+d H d x d$

Here $d$ is the point defect under consideration, and $x_{d}$ its atomic concentration. $H d$ is the formation enthalpy of the defect.

The energy change is defined as:

$$
\left.E X N i \rightarrow A l=E\left(\mathrm{Ni}_{24} \mathrm{Al}_{7} \mathrm{X}\right)+E\left(\mathrm{Ni}_{23} \mathrm{AlAl}{ }_{8}\right)-E\left(\mathrm{Ni}_{23} \mathrm{XAl}\right)_{8}\right)-E\left(\mathrm{Ni}_{24} \mathrm{Al}_{8}\right)
$$

[2]

When this quantity is negative, the atom preferentially occupies the Al site, while when it is positive the atom occupies either the Ni site or both sites. More precisely,

a) $E X N i \rightarrow A l<0$, X occupies Al site

b) $E X N i \rightarrow A l>H A l N i+H N i A l=1.12 \mathrm{eV}[7]$, X occupies Ni site

c) $0<E X N i \rightarrow A l<H A l N i+H N i A l, X$ atoms can occupy both sites

Some dopants of interest for $\mathrm{Ni}_{3} \mathrm{Al}$ include $\mathrm{B}$ for enhanced mechanical strength [12, 13], $\mathrm{Cr}$ for improved oxidation resistance [4, 13], and Ce for both better mechanical strength and oxidation resistance [12, 14]. Zr provides strengthening and resistance to creep [15,16] and La has shown to improve resistance to oxidation and hot corrosion [13]. While it is established that Ce forms a second phase $\mathrm{CeNi}_{5}$ at concentrations exceeding $0.63 \mathrm{wt} \% \mathrm{Ce}$ [17], Ce addition contributes to the desired properties. However, it is a rare earth element and a challenge exists to replace $\mathrm{Ce}$ with a non-rare earth element while maintaining the desired properties. While $\mathrm{Ce}$ and $\mathrm{Cr}$ are substitutional, B occupies interstitial voids owing to its small size. As a result, the replacement of $\mathrm{Ce}$ with $\mathrm{Cr}$ and $\mathrm{B}$ for oxidation resistance and mechanical strength is not possible, due to their different site occupancies. This defines the challenge that is addressed by this paper, namely to assess the change in properties that result for changes to both composition and site occupancy. 
The following doping chemistries and sites discussed here are: undoped $\mathrm{Ni}_{3} \mathrm{Al}, \mathrm{Ce}, \mathrm{La}, \mathrm{Cr}$ and $\mathrm{Zr}$ doped on the $\mathrm{Ni}$ site, $\mathrm{Ce}, \mathrm{La}, \mathrm{Cr}$ and $\mathrm{Zr}$ doped on $\mathrm{Al}$ site, and $\mathrm{B}$ doped on tetrahedral interstitial and octahedral interstitial voids. Beyond the properties of interest, these chemistries were selected so that their additional differences such as size differences and periodic table groups are integrated into the informatics analysis. For each of these compounds, the electronic Density of States (DOS) and bulk modulus $\left(B_{m}\right)$ were calculated via Density Functional Theory (DFT). An informatics analysis is then applied to the DOS spectra for assessing the possible compound chemistries that can exist, comparing behavior of different dopant chemistries, and linking dopant site occupancies with mechanical behavior. We have previously applied an informatics analysis to the DOS spectra [1-3], although those previous works did not consider site occupancies. Other approaches combining informatics algorithms with electronic structure calculations sought to accelerate the existing electronic structure calculations so that data can be calculated in a high-throughput manner, and to search for appropriate crystal structures or structure determination from the significantly increased knowledge-base [18-20]. An alternate informatics approach for predicting the bulk and shear moduli has recently been demonstrated by de Jong et al [21-22], where a massive number of property values were predicted based on series of descriptors including formation energy, volume-per-atom, other elemental properties, and relative elemental positioning within the periodic table.

By employing the entire DOS spectra as we do here, the span of reversible property descriptors are theoretically included without any prior assumptions. As the DOS represents all electronic interactions of a system, it should contains information on all electronic properties [23-25]. However, the understanding of how these properties are captured by the DOS is not well understood. One example of a property which is known to be at least qualitatively represented within the DOS spectra is bulk modulus [26], where the difference between Fermi energy and antibonding-bonding transition correlates to $B_{m}$. Some physical explanation of the connection between $B_{m}$ and DOS spectra can thus be provided, although it remains qualitative and limited. Building on this concept is one of the primary objectives in linking DOS spectra with an informatics approach. By contrast, we use informatics approaches to relate patterns in the DOS spectra to the bonding of alloy systems and the corresponding properties.

$\mathrm{Cr}$ has previously been shown to have an Al site preference via calculation of $E_{X}{ }^{\mathrm{Ni}-\mathrm{Al}}[5-10]$. Calculation of this quantity for $\mathrm{Ce}$ substitution has not been reported. To predict this site occupancy, we must understand why $\mathrm{Cr}$ occupies the $\mathrm{Al}$ site and not the Ni site. In addition to assessing the calculations at $0 \mathrm{~K}$, we perform experimental analysis of $\mathrm{Ni}_{3} \mathrm{Al}-\mathrm{Cr}$ to ensure that the modeling of site occupancy holds at higher temperatures. While the focus of this paper is limited to site preference, we also recognize that the concentration of defects is critical to consider since the properties discussed here are largely dependent on the final equilibrium state of the system. Future work will address this added layer of consideration by including chemical potentials to describe the thermodynamic interactions between the $y$ and $y$ ' phases. This paper introduces an informatics driven approach for extracting previously undefined chemistryelectronic structure-property correlations, even though the systems considered have been extensively studied previously. We discuss how this added layer of information which is not available solely from the unprocessed DFT output allows us to accelerate the design of new Nibase alloys for specific applications.

\section{Methods}




\subsection{Density Functional Theory Calculations}

The supercell consisted of $2 \times 2 \times 2$ unit cells of $\mathrm{Ni}_{3} \mathrm{Al}$, shown in Fig.1 (a). Cr, $\mathrm{Zr}$, La and $\mathrm{Ce}$ dopants were placed in either the Al or Ni site (Fig. 1 (b) and (c)), while B was placed in the tetrahedral and octahedral void (Fig. 1(c) and (d)). Cr, Zr, La and Ce were varied with concentrations of 3.125 at $\%, 6.25$ at\% and 9.375 at\%, which corresponds to 1, 2 and 3 dopant atoms substituting either $\mathrm{Ni}$ or $\mathrm{Al}$ site in $\mathrm{Ni}_{3} \mathrm{Al}$. For $\mathrm{B}$, the concentration used was 3.03 at $\%$. The concentrations were chosen keeping in mind the compositions used in superalloys [27].

The Vienna Ab initio Simulation Package (VASP) [28-31] was used to perform the density functional theory (DFT) calculations. The exchange-correlation functional by Perdew, Burke and Ernzerhof was employed within generalized gradient approximation (PBE-GGA) [32, 33]. Projector Augmented Wave (PAW) scheme [34] was used to account for the core electrons, while the energy cut-off for the plane wave basis was chosen to be $350 \mathrm{eV}$, which was determined to be a suitable value. A Monkhorst-Pack [35] K-mesh of $6 \times 6 \times 6$ was used. All calculations were spin-polarized and the atoms were relaxed with a convergence criteria of $10^{-7}$ $\mathrm{eV}$ for energy and $10^{-3} \mathrm{eV} / \AA$ for the forces on atoms. The elastic constants were then extracted from the stress-strain relationship by straining the relaxed structures to obtain $C 11, C 12$, and

$C 44$, where $\quad C 11=(C 11+C 22+C 33) / 3, \quad C 12=(C 12+C 13+C 23) / 3 \quad$ and $C 44=(C 44+C 55+C 66) / 3$. The convergence achieved was within an error bar of $8 \mathrm{GPa}$ except in the case of $\mathrm{Ce}$-doped $\mathrm{Ni}_{3} \mathrm{Al}$ on the $\mathrm{Ni}$-site where the error bar range is 10-15 GPa. Complete details of the elastic constant calculations and the convergence achieved are provided in our prior report [7]. The density of states (DOS) was calculated with the energy convergence criterion increased to $10^{-8} \mathrm{eV}$ and a Monkhorst-Pack K-point mesh of $9 \times 9 \times 9$ for both pure and doped $\mathrm{Ni}_{3} \mathrm{Al}$ systems. The Fermi-energy was shifted to $0 \mathrm{eV}$ for all the calculations for consistency. 


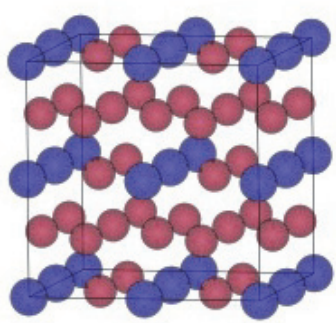

(a)

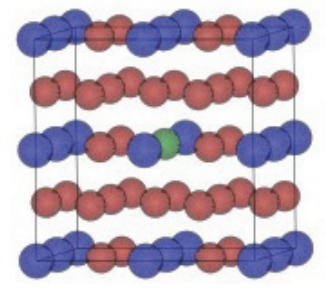

(b)

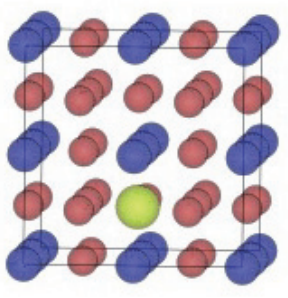

(c)

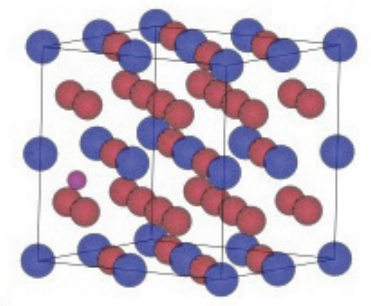

(d)

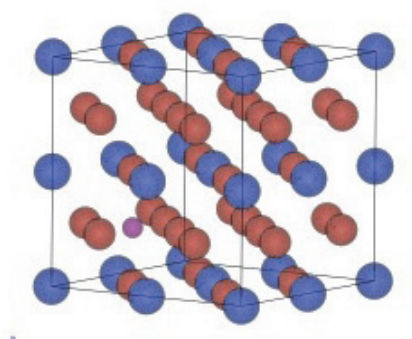

(e)

Figure 1. Crystal structure and site substitution for (a) $L_{1} 1_{2}-N i_{3} A l$, which serves as input into the DFT modeling; (b) Cr atom substituting for an Al atom; (c) Ce atom substituting for Ni atom, B atom occupying $(d)$ a tetrahedral void, and (e) an octahedral void.

\subsection{Informatics}

To identify the underlying governing features within the DOS spectra, we employed the entire DOS spectra to quantitatively analyze the DOS without any prior assumptions by treating the DOS as spectral data, building on our prior work in analyzing spectral data [36-37]. We apply eigennvalue decomposition techniques such as principal component analysis (PCA) [36-41]. As we have demonstrated in our earlier studies [1-3,36-38], this permits one to identify the right projection of data from which meaningful features associated with the DOS can be identified. Partial Least Squares (PLS) were further applied to this high dimensional data space [42-50]. These were used in a predictive informatics mode to link the DOS spectra with elastic constants. By applying PCA to the DOS spectra, the strongest patterns in the data can be identified in a limited number of dimensions.

PCA operates by performing an eigenvector decomposition of the data. As such, the principal components (PCs) capturing the most information are associated with the largest eigenvalues of the covariance matrix and their corresponding eigenvectors. The original data is decomposed into two matrices of interest for this paper: the scores and loadings. The loadings and scores matrices contain the principle patterns within the DOS curves and the scaling of those patterns to create the final DOS curve, respectively. In PLS the training data is converted to a data matrix with orthogonalized axes, which are based on capturing the maximum amount of information in fewer dimensions. The relationships discovered in the training data can be applied to a test dataset based on a projection of the data onto a high-dimensional hyperplane within the orthogonalized axis-system. Typical linear regression models do not properly account for the colinearity between the descriptors, and as a result the isolated impact of each descriptor on the property cannot be accurately known. 
The ability to predict properties of alloy systems from an input of DOS requires the integration of PCA and PLS. This approach represents a hybrid informatics approach because properties are determined as a summation of the PLS coefficients (the prediction coefficients of the PC scores values on the elastic constants) and the PCA weightings (ie. Property $=f[\Sigma$ (Component of PLS result * Component of PCA result]) as opposed to isolating the components (ie. Property = $\mathrm{f}\left[\Sigma\left(\right.\right.$ Component of PLS result) ${ }^{*} \Sigma$ (Component of PCA result)]. That is, in the final development of an equation, the PLS and PCA components of the analysis cannot be separately extracted. This hybrid capacity of the approach is utilized in this paper to connect elastic constants with the corresponding DOS spectra.

\subsection{Experiment}

A Ni-Al-Cr superalloy crystal with nominal composition of Ni-75.7 Al-16.7 Cr-7.5 at \% was grown by the directional Bridgman technique. The as-cast sample was then annealed at $1250{ }^{\circ} \mathrm{C} \pm 10$ ${ }^{\circ} \mathrm{C}$ for 14 days in He environment. TEM specimens were prepared by conventional mechanical polishing followed by low energy ion milling recipe. Atomic resolution EDS analysis was performed with a probe-corrected FEI Titan G2 60-300 KV S/TEM equipped with a highbrightness Schottky field emission gun, operated at $200 \mathrm{kV}$. The convergence angle and HAADF detector inner semi-angle are $21 \mathrm{mrad}$ and $77 \mathrm{mrad}$, respectively. The EDS elemental maps were acquired with a beam current of $\sim 0.1 \mathrm{nA}$. A 5-pixel smoothing filter was applied to the EDS elemental maps during post-processing to reduce noise using Bruker esprit software package.

\section{Results}

DFT calculations were performed for undoped $\mathrm{Ni}_{3} \mathrm{Al}, 0.03 \% \mathrm{~B}$ doped in interstitial voids, $\mathrm{Ni}_{24} \mathrm{Al}_{8-}$ ${ }_{x} \mathrm{Cr}_{\mathrm{x}}, \mathrm{Ni}_{24-x} \mathrm{Al}_{8} \mathrm{Cr}_{\mathrm{x}}, \mathrm{Ni}_{24} \mathrm{Al}_{8-\mathrm{x}} \mathrm{Zr}_{\mathrm{x}}, \mathrm{Ni}_{24-x} \mathrm{Al}_{8} \mathrm{Zr}, \mathrm{Ni}_{24} \mathrm{Al}_{8-\mathrm{x}} \mathrm{Ce}_{\mathrm{x}}, \mathrm{Ni}_{24-x} \mathrm{Al}_{8} \mathrm{Ce}, \mathrm{Ni}_{24} \mathrm{Al}_{8-\mathrm{x}} \mathrm{La}_{\mathrm{x}}$ and $\mathrm{Ni}_{24-x} \mathrm{Al}_{8} \mathrm{La}_{\mathrm{x}}$, for $x=1,2,3$. The output of the calculations were DOS spectra (Fig. 2) and bulk moduli (Table 1). From visual inspection of the spectra, the change due to site occupancy is not significantly evident and emphasizes the need to apply manifold learning analysis. The doping of B causes an increase in DOS at low energies, while the addition of Ce causes significant increase above the Fermi energy $\left(E_{F}=0 \mathrm{eV}\right)$ due to the introduction of f-states. However, these visual interpretations do not provide any information on the correlation between the change in DOS spectra with changing chemistry and the change in elastic properties. Identifying those correlations provides a more critical design guideline for modifying dopant chemistry. 

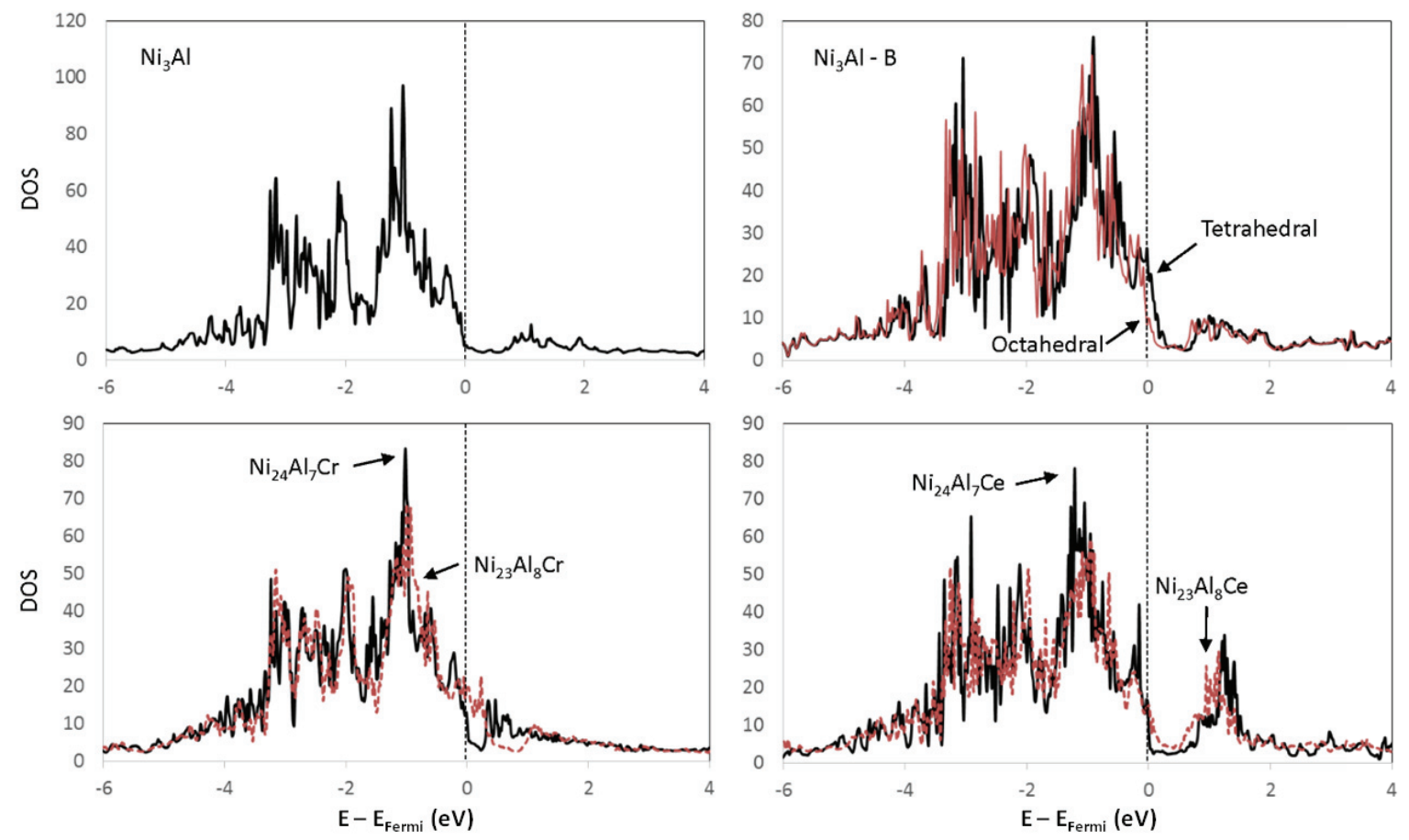

Figure 2. Input DOS for the informatics analysis. While changes in the spectra are evident with changing dopant chemistry, the changes due to site occupancy are much more subtle. Informatics is applied to these spectra so that hidden features which control site occupancy energy are evident and possible chemistries are rapidly determined.

Table 1. DFT calculated elastic properties [7]. Using these properties for dopant chemistry and site, correlations between mappings of DOS spectra and mechanical 
properties can be made, developing a quantitative structure-property relationship for new compounds.

\begin{tabular}{|c|c|c|c|c|}
\hline & $C$ & $C$ & $C$ & $B$ \\
\hline Ni3Al & 246 & 155 & 130 & 185.33 \\
\hline $\operatorname{Ni3Al(B)*~}$ & 255 & 148 & 118 & 183.67 \\
\hline $\mathrm{Ni3Al(B)}{ }^{\#}$ & 255 & 149 & 118 & 184.22 \\
\hline $\mathrm{Ni}_{24} \mathrm{Al}_{7} \mathrm{Cr}$ & 252 & 154 & 131 & 186.67 \\
\hline $\mathrm{Ni}_{24} \mathrm{Al}_{6} \mathrm{Cr}_{2}$ & 262 & 156 & 137 & 191.11 \\
\hline $\mathrm{Ni}_{24} \mathrm{Al}_{5} \mathrm{Cr}_{3}$ & 266 & 156 & 137 & 192.56 \\
\hline $\mathrm{Ni}_{23} \mathrm{Al}_{8} \mathrm{Cr}$ & 233 & 152 & 125 & 179.33 \\
\hline $\mathrm{Ni}_{22} \mathrm{Al}_{8} \mathrm{Cr}_{2}$ & 239 & 161 & 125 & 186.78 \\
\hline $\mathrm{Ni}_{21} \mathrm{Al}_{8} \mathrm{Cr}_{3}$ & 232 & 152 & 121 & 178.78 \\
\hline $\mathrm{Ni}_{24} \mathrm{Al}_{7} \mathrm{Zr}$ & 246 & 152 & 123 & 183.33 \\
\hline $\mathrm{Ni}_{24} \mathrm{Al}_{6} \mathrm{Zr}_{2}$ & 243 & 145 & 119 & 177.89 \\
\hline $\mathrm{Ni}_{24} \mathrm{Al}_{5} \mathrm{Zr}_{3}$ & 248 & 147 & 111 & 180.89 \\
\hline $\mathrm{Ni}_{23} \mathrm{Al}_{8} \mathrm{Zr}$ & 225 & 149 & 115 & 174.67 \\
\hline $\mathrm{Ni}_{22} \mathrm{Al}_{8} \mathrm{Zr}_{2}$ & 220 & 142 & 104 & 167.89 \\
\hline $\mathrm{Ni}_{21} \mathrm{Al}_{8} \mathrm{Zr}_{3}$ & 207 & 138 & 95 & 160.67 \\
\hline $\mathrm{Ni}_{24} \mathrm{Al}_{7} \mathrm{Ce}$ & 235 & 141 & 113 & 172.44 \\
\hline $\mathrm{Ni}_{24} \mathrm{Al}_{6} \mathrm{Ce}_{2}$ & 236 & 141 & 97 & 202.20 \\
\hline $\mathrm{Ni}_{24} \mathrm{Al}_{5} \mathrm{Ce}_{3}$ & 229 & 135 & 85 & 207.19 \\
\hline $\mathrm{Ni}_{23} \mathrm{Al}_{8} \mathrm{Ce}$ & 221 & 132 & 104 & 190.44 \\
\hline $\mathrm{Ni}_{22} \mathrm{Al}_{8} \mathrm{Ce}_{2}$ & 213 & 126 & 80 & 190.44 \\
\hline $\mathrm{Ni}_{21} \mathrm{Al}_{8} \mathrm{Ce}_{3}$ & 178 & 110 & 70 & 190.44 \\
\hline $\mathrm{Ni}_{24} \mathrm{Al}_{7} \mathrm{La}$ & 229 & 134 & 109 & 165.67 \\
\hline $\mathrm{Ni}_{24} \mathrm{Al}_{6} \mathrm{La}_{2}$ & 213 & 124 & 92 & 153.44 \\
\hline $\mathrm{Ni}_{24} \mathrm{Al}_{5} \mathrm{La}_{3}$ & 198 & 114 & 79 & 142.11 \\
\hline $\mathrm{Ni}_{23} \mathrm{Al}_{8} \mathrm{La}$ & 213 & 137 & 102 & 162.22 \\
\hline $\mathrm{Ni}_{22} \mathrm{Al}_{8} \mathrm{La}_{2}$ & 198 & 129 & 88 & 151.89 \\
\hline $\mathrm{Ni}_{21} \mathrm{Al}_{8} \mathrm{La}_{3}$ & 212 & 97 & 59 & 135.22 \\
\hline
\end{tabular}

The informatics analysis was performed on 27 compounds, with the result shown in Fig. 3. Each point within the DOS spectra was considered as a separate descriptor, so that the dimensionality of the problem was on the order of $10^{3}$. Sampling a larger number of points from the DOS spectra results in a higher resolution result, but also a less robust result as specific features may be over-fit as opposed to capturing the general and physically significant trends. Further, the sensitivity to exact DFT parameters is reduced, and our objective of developing a generalized approach is enhanced. Therefore, after performing multiple informatics simulations to compare accuracy versus robustness, this number of points was identified as capturing the same results as larger descriptor sets while enhancing the potential robustness. The principal components (PC1 and PC2) provide two new uncorrelated descriptors of the data, and the 
scores values provide a parameterization of the DOS spectra which is based on maximizing information extraction. Relationships in the data are then defined in terms of Euclidean distance and trajectory. PC1 captures changes in the DOS due to Ce doping, as evidenced by $\mathrm{Ce}$ increase along PC1, while other doping chemistries have little change along this axis. PC2 captures changes in DOS due to other dopings with La having the largest impact. This demonstrates the impact in rare earth additions. Doping $B$ in the octahedral site has a larger effect as compared to doping in the tetrahedral site, as the compound with $B$ in the tetrahedral site sits closer to pure $\mathrm{Ni}_{3} \mathrm{Al}$.

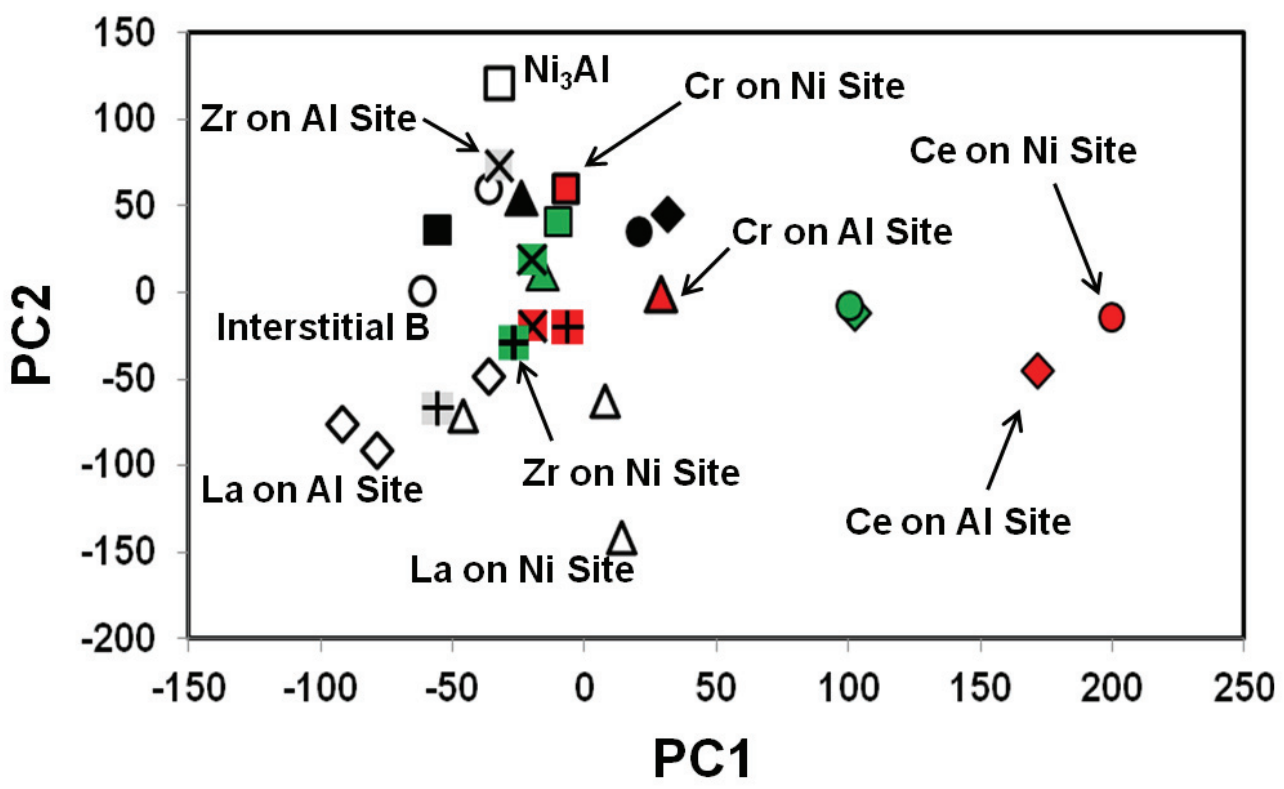

Figure 3. Principal component projection showing the correlations associated with site occupancy and site chemistry. Black/gray filled shapes correspond to 3.125 at\% dopant, green is 6.25 at\% dopant and red filled shapes are 9.375 at\% dopant concentration. Filled squares are $\mathrm{Cr}$ on Ni site, triangles are $\mathrm{Cr}$ on $\mathrm{Al}$ site, circles are $\mathrm{Ce}$ on Ni site, diamonds are $\mathrm{Ce}$ on Al site, ' $X$ ' are Zr on Al site and '+' are Zr on Ni site. Unfilled diamonds are La on Al site, unfilled triangles are La on Ni site, and unfilled circles are $B$ on interstitial sites. From this we identify $\mathrm{Ce}$ as occupying either site, Cr occupying only Al site, $\mathrm{Zr}$ occupying only Al site, and La not having a strongly stable additional configuration. As an example, the reason $\mathrm{Cr}$ does not occupy Ni site is described by the DOS, as evidenced by little relationship between Cr content and $\mathrm{PC}$ values, while $\mathrm{Cr}$ on $\mathrm{Al}$ site forms a clear trend from undoped Ni3Al to increasing $\mathrm{Cr}$ content. This interpretation can be applied to each chemistry, with stability defined by clear trends associated with additive composition.

The trajectory in this map with increasing dopant concentration provides an explanation of the energetics of site substitution. The interpretation of site occupancy is that the increase of dopant continues to make the material more dissimilar to $\mathrm{Ni}_{3} \mathrm{Al}$. However, if it is not energetically favorable for the atom to be located on that site, the DOS will not change in such a consistent manner. When looking at $\mathrm{Cr}$ addition, we find that the alloy becomes less like $\mathrm{Ni}_{3} \mathrm{Al}$ 
in a consistent manner with concentration of $\mathrm{Cr}$ on the $\mathrm{Al}$ site. However, when adding $\mathrm{Cr}$ on the $\mathrm{Ni}$ site, there is no clear pattern, as demonstrated by the arced trajectory. The lack of clear systematics represents the unfavorable energetics of $\mathrm{Cr}$ addition to the Ni site. Building on this interpretation that the trajectory between PC values and dopant chemistry captures the defect formation energy, we predict that $\mathrm{Ce}$ is able to occupy either site due to the linear trajectories of both site substitutions. Note that the relationships with chemistry and site occupancy cannot be identified from the raw DOS spectra, and $\mathrm{Cr}$ on the Ni site would not be identified as an outlier chemistry without informatics. As the informatics analysis is extracting the strongest physics based trends within the DOS spectra, it is fairly insensitive to minor changes in the DOS which would result from variation of DFT parameters, resulting in a robust generalized approach. Further, the predictions from the data mining are in good agreement with the training cases of no occupancy of $\mathrm{Cr}$ and $\mathrm{Zr}$ on $\mathrm{Ni}$ site, strengthening the argument that the DFT calculation mesh and supercell sizes were sufficient as an input into the informatics model.

To test the predictions for $\mathrm{Cr}$, we experimentally assessed the site substitution via EDS. The typical microstructure of the ternary Ni-Al-Cr compound consisted of a disordered (fcc structure) $\mathrm{Y}$-matrix with spherical ordered $\left(\mathrm{L} 1_{2}\right.$ structure) $\mathrm{Y}$ '-precipitates. EDS was acquired in the ordered region of the $\mathrm{Ni}-\mathrm{Al}-\mathrm{Cr}$ sample (Fig. 4), demonstrating that $\mathrm{Cr}$ sits preferentially at the Al sites in $\mathrm{Ni}_{3} \mathrm{Al}$. This result is consistent with experimental observations in Ref. [51] and agrees with our prediction. While the DFT calculations are performed for $0 \mathrm{~K}$, the experimental agreement indicates that the DFT and informatics predictions are reasonable for materials at operational temperatures. Therefore, we expect that the prediction of $\mathrm{Ce}$ site occupancy is valid at operational temperatures as well.
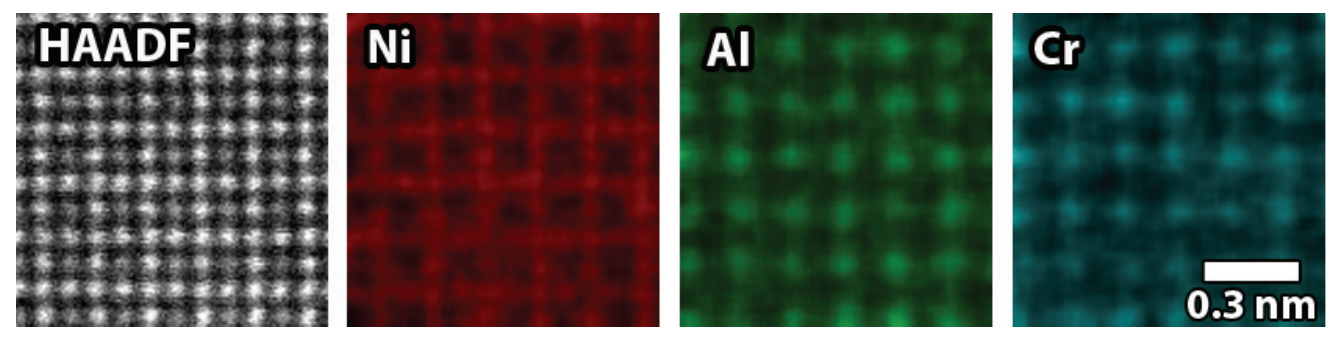

Figure 4. EDS elemental mapping of $\mathrm{Ni}_{3} \mathrm{Al}-\mathrm{Cr}$ in the $<100>$ projection. This result shows $\mathrm{Cr}$ having preferred $\mathrm{Al}$ substitution in $\mathrm{NI}_{3} \mathrm{Al}$, agreeing with our informatics-based prediction.

As a further testing of the relationship of our mapping with bulk modulus, we compare the change in PC space based only on DOS input (Figure 3) with the change in moduli listed in Table 1. From this the following results are found: (i) $\mathrm{Cr}$ prefers $\mathrm{Al}$ site and modulus increases with addition of $\mathrm{Cr}$ on $\mathrm{Al}$ site and decreases with addition on Al site; (ii) Zr prefers Al site and modulus has larger decrease with $\mathrm{Zr}$ added to $\mathrm{Ni}$ site; (iii) Ce can occupy either site and modulus does not decrease with $\mathrm{Ce}$ addition to either site; and (iv) La decreases stability when added to either site and modulus decreases significantly with increased Al to either site. Therefore, we have demonstrated here that by mining only the DOS, we predict the site occupancy (prediction matching experiment) and also the relationship of site occupancychemistry-bulk modulus relationship (prediction matching DFT). 


\section{Discussion}

As was shown in Figs. 3 and 4 (and as further highlighted in Fig. 5a), the $\mathrm{Cr}$ atoms preferr Al site occupancy. While this conclusion could be made solely from DFT calculations, we apply informatics in order to extract signature of the DOS corresponding to simultaneous change in chemistry and property. This added information which is not readily available from solely the DFT output accelerates alloy design by reducing the complex DFT output to a limited number of key features or parameters. By interrogating the corresponding eigenspectra from the spectral decomposition (Fig. 5b), we can extract these signatures. The eigenspectra define the primary patterns within the DOS spectra based on maximizing the information captured. As the DOS spectra were mean centered prior to analysis, if each respective eigenspectra is multiplied by the corresponding scores parameter and then summed, the result is the initial mean centered spectra. Therefore, those features in the eigenspectra with the most significant magnitudes are the most important for defining the axes of Fig. 5a. For PC1, the peak corresponding with the fstates is the most significant. This matches with the interpretation that PC1 is capturing primarily effects due to Ce doping, as $\mathrm{Ce}$ is the only element considered here with f-electrons. For purposes of defining site occupancy in $\mathrm{Ni}_{3} \mathrm{Al}-\mathrm{Cr}, \mathrm{PC} 2$ is the critical axis to consider. When increasing the $\mathrm{Cr}$ concentration at the Al sites, the PC2 value continues to increase. However, when increasing the $\mathrm{Cr}$ concentration at the $\mathrm{Ni}$ sites, the PC2 value does not change significantly. To understand why $\mathrm{Cr}$ occupies the Al site, we must identify why PC2 does not change with increasing $\mathrm{Cr}$ content in terms of the DOS spectra.
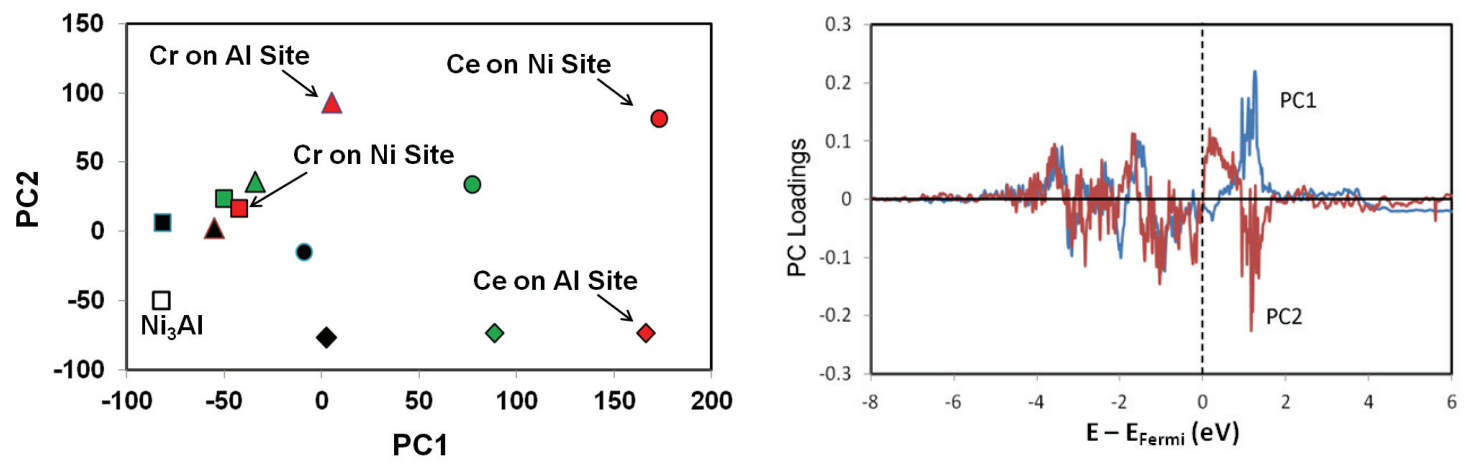

Figure 5. This analysis corresponds with the analysis of Figure 3 but showing fewer compounds for clarity (a) and for showing how to interpret the loadings spectra (b). Increased DOS values at energies corresponding to positive loadings will increase the respective PCs, while the inverse case is true for negative loadings values. PC1 primarily captures changes due to increasing Ce composition, with the primary feature of the PC1 eigenspectra corresponding to the f-state. PC2 defines why Cr occupies only Al sites and also describes the change in bulk modulus.

To link this mapping with mechanical properties of site occupancy, a model was developed for predicting bulk modulus as a function of PC values. The resulting equation was: $B_{m}=f(-$ $\left.0.003^{*} \mathrm{PC} 1-0.17^{*} \mathrm{PC} 2\right)$. This modulus value was obtained by considering only doped systems, and not including $\mathrm{Ni}_{3} \mathrm{Al}$. Since the coefficient of both PC1 and PC2 is negative (ie. -0.003 and 0.17 ), $B$ is inversely correlated with both parameters. Also, given the relative values of the coefficients, we find that PC2 is more significant for determining $B_{m}$, i.e. a change in PC2 will 
affect $B_{m}$ more than an equivalent change in PC1. From this result, we identify that increasing Ce composition on the $\mathrm{Ni}$ site has a larger effect on $\mathrm{B}_{\mathrm{m}}$ than increasing Ce composition on the Al site. This is not evident when looking at Table 1. This map therefore integrates dopant chemistry, site substitution, and mechanical property. The findings associated with Ce doping are that Ce can occupy either $\mathrm{Ni}$ or $\mathrm{Al}$ site, while the modulus is more sensitive to Ce on $\mathrm{Ni}$ site.

As there are multiple features present in the PC2 spectrum, it is instructive to compare with the relevant DOS spectra (Fig. 6). To define the change in DOS spectra with Cr concentration, the DOS values at each energy for $\mathrm{Ni}_{23} \mathrm{Al}_{8} \mathrm{Cr}$ were subtracted from those for $\mathrm{Ni}_{21} \mathrm{Al}_{8} \mathrm{Cr}_{3}$, and also for $\mathrm{Ni}_{24} \mathrm{Al}_{7} \mathrm{Cr}$ from $\mathrm{Ni}_{24} \mathrm{Al}_{5} \mathrm{Cr}_{3}$. In this representation of the DOS, we identify two notable peaks for $\mathrm{Cr}$ in the $\mathrm{Al}$ site. These two peaks, centered at approximately $-1.7 \mathrm{eV}$ and $0.2 \mathrm{eV}$ (where this energy is defined as energy minus $E_{F}$ ) correspond to high intensity regions in the loadings spectra, which means that changes in the DOS at these energies will impact the PC2 values. Therefore, the difference in DOS at these energies results in a linear relationship between PC2 value and $\mathrm{Cr}$ composition for $\mathrm{Al}$ substitution and the lack of a relationship for Ni substitution. The reason that $\mathrm{Cr}$ goes to the $\mathrm{Al}$ site in $\mathrm{Ni}_{3} \mathrm{Al}$ is because the $\mathrm{DOS}$ at $-1.7 \mathrm{eV}$ and $0.2 \mathrm{eV}$ is insensitive to $\mathrm{Cr}$ addition at the $\mathrm{Ni}$ site but is sensitive to $\mathrm{Cr}$ addition at the Al site. In Figs. 3 and 5 , we found that site occupancy is based on linear relationship between PC values and dopant concentration. As the $\mathrm{Cr}$ doped samples change along PC2, in order for the relationship to remain linear, the DOS must vary at energies corresponding to those with highest loadings values. For $\mathrm{Cr}$ substituted on $\mathrm{Al}$ site, we see large difference in DOS with increasing $\mathrm{Cr}$ content at the circled energies. However, for $\mathrm{Cr}$ substituted on $\mathrm{Ni}$ site, there is little change in DOS at those energies. The lack of changing DOS at those two energy ranges with increasing $\mathrm{Cr}$ content is the reason that $\mathrm{Cr}$ does not occupy the Ni site.

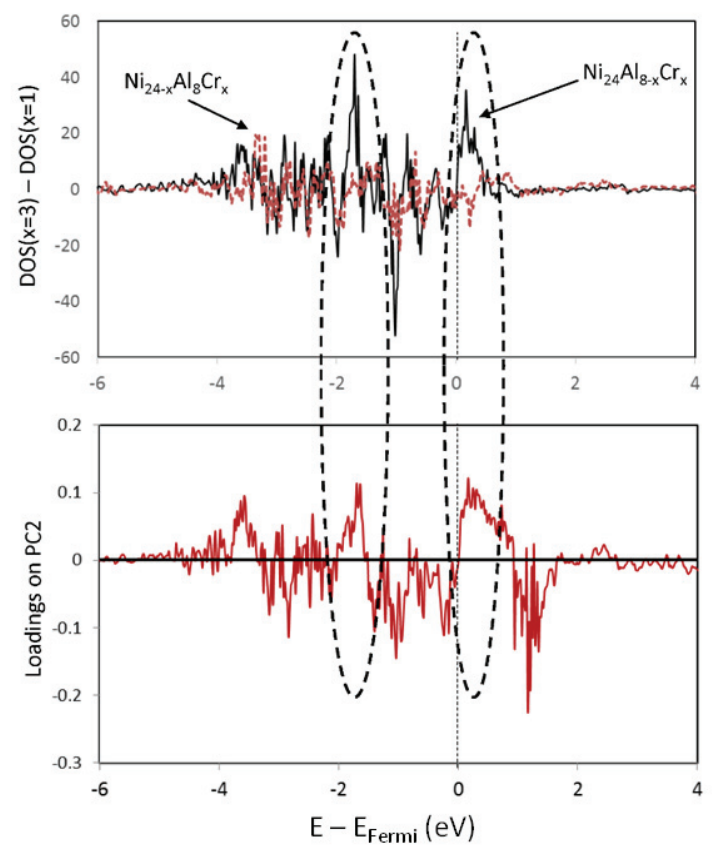

Figure 6. Comparing eigenspectrum with DOS as function of changing site occupancy for understanding why $\mathrm{Cr}$ occupies $\mathrm{Al}$ site. The circled regions correspond with the energies responsible for the preferred Al site substitution for $\mathrm{Cr}$. 
In order to simplify the visualization of the chemistry-property correlations, we developed a dendrogram which defines this similarity (Figure 7). The input was the change in PC space with increased dopant concentration and the resulting elastic constants. This dendrogram therefore defines similarity between compounds based solely on the chemistry-property correlation as derived from the DOS. As the connection of branches moves up in the dendrogram, the correlation decreases. For example, we find that the similarity in chemistry-property relationship is highest for $\mathrm{Cr}$ on an $\mathrm{Al}$ site and $\mathrm{Ce}$ on an $\mathrm{Al}$ site. These are next most similar to $\mathrm{Zr}$ on a Ni site. Following this logic, $\mathrm{Zr}$ shows the least site occupancy effect of any dopant, with La also having a small occupancy effect. In terms of identifying chemical substitutes for rare earth elements, we find that $\mathrm{Cr}$ on a $\mathrm{Ni}$ site is on the same branch as La on either site and $\mathrm{Ce}$ on a $\mathrm{Ni}$ site. This therefore provides a design guideline for replacing rare earth elements, although as discussed $\mathrm{Cr}$ preferentially occupies the Al site. However, the strategy for replacing the rare earth element with minimal impact on property is to identify an element with similar characteristics to $\mathrm{Cr}$ and which may occupy a $\mathrm{Ni}$ site, or else to identify a combination of elements to modify the couple critical signatures of the DOS associated with $\mathrm{Cr}$ site occupancy which we identified. Future work will incorporate elements which preferentially occupy Ni site, based on this identified chemical modification strategy.

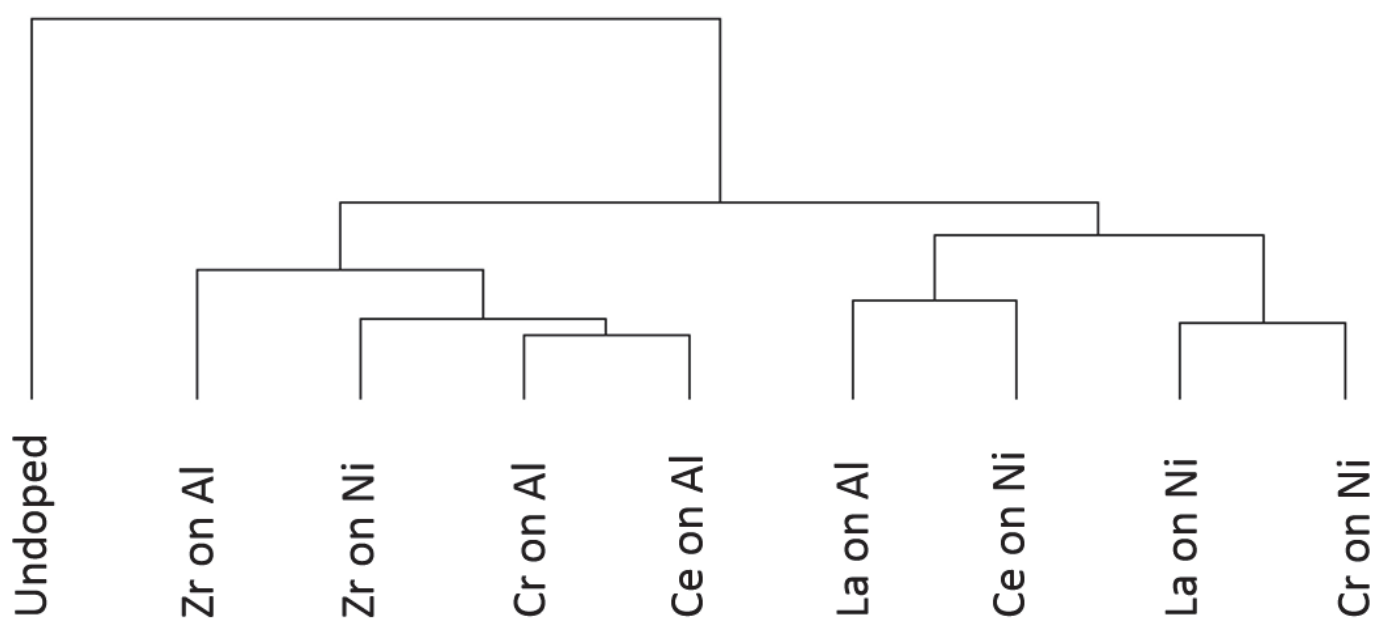

Figure 7. Dendrogram comparing similarity between dopants at specific sites in terms of the effect on the DOS spectra and the elastic moduli. The inputs into developing the dendrogram are the change in PC positions in Figure 3 with added dopant concentration, and the resulting elastic constants. The lines connecting branches nearer the bottom of the figure indicate higher degree of correlation (eg. Cr on Al site and $\mathrm{Ce}$ on Al site are most correlated, while there is minimal correlation between undoped $\mathrm{Ni}_{3} \mathrm{Al}$ and doped $\mathrm{Ni}_{3} \mathrm{Al}$. From this, we find that $\mathrm{Cr}$ has the most similarity to rare earth dopants, while $\mathrm{Zr}$ and La are the least impacted by site occupancy. 


\section{Conclusions}

In this paper, we developed an approach for identifying the signatures of the DOS which capture the correlation between site occupancy energetics and elastic moduli. From these signatures, we assessed the similarity between different chemistries of $\mathrm{L}_{1}{ }_{2}-\mathrm{Ni}_{3} \mathrm{Al}$. As an example of the application of this work for chemical design, we assessed the similarity in the chemistry-property correlation between elements in order to identify potential rare earth replacements. We find $\mathrm{Cr}$ on the $\mathrm{Ni}$ site as the most similar to the rare earth containing compounds, although $\mathrm{Cr}$ on the $\mathrm{Al}$ site is also similar to $\mathrm{Ce}$ on the Al site. From our analysis, we identified that $\mathrm{Cr}$ preferentially occupies the Al site. In order to understand what features of the DOS spectra to modify through doping in order to leverage the similarity with rare earth elements, we extracted the key signatures at -1.7 and $0.2 \mathrm{eV}$ for $\mathrm{Cr}$ doping. This provides guidelines for further chemical modification for targeted design. This work has demonstrated how the application of data dimensionality reduction methods can serve as an accelerated feature selection approach to interrogate DOS spectra coupled to a mechanistic interpretation of linking chemistry and site occupancy to design alloy chemistries with targeted mechanical properties.

\section{Acknowledgements}

We gratefully acknowledge support of this work under Air Force Office of Scientific Research (AFOSR) Grant No. FA9550-12-1-0456 and NSF Grant No. DMR-13-07811. SB and KR acknowledge support from NSF DIBBs Award Number ACl-1640867. KR also acknowledges support from the Erich Bloch Endowed Chair at the University at Buffalo-State University of New York. All the DFT calculations were performed using computational resources provided by the University of Florida Research Computing (http://researchcomputing.ufl.edu/). AAO and JML acknowledge the use of the Analytical Instrumentation Facility (AIF) at North Carolina State University, which was supported by the State of North Carolina and the National Science Foundation.

\section{References}

[1] S. R. Broderick and K. Rajan, Eigenvalue decomposition of spectral features in density of states curves, Europhys. Lett. 95 (2011) 57005.

[2] S. R. Broderick, H. Aourag, and K. Rajan, Classification of oxide compounds through datamining density of states spectra, J. Am. Ceram. Soc. 94, (2011) 2974.

[3] S. R. Broderick, H. Aourag, and K. Rajan, Data mining density of states spectra for crystal structure classification: An inverse problem approach, Stat. Anal. Data Min. 6, (2009) 353-360.

[4] R.C. Reed, The Superalloys Fundamentals and Applications, Cambridge University Press, Cambridge, 2006.

[5] D.E. Kim, S.L. Shang, Z.K. Liu, Effects of alloying elements on elastic properties of Ni3Al by first principles calculations, Intermetallics, 18 (2010) 1163-1171.

[6] Q. Wu, S. Li, Alloying element additions to Ni3Al: Site preferences and effects on elastic properteis from first-principles calculations, Comput. Mater. Sci., 53 (2012) 436-443.

[7] A. Kumar, A. Chernatynskiy, M. Hong, S.R. Phillpot, S.B. Sinnott, An ab initio investigation of the effect of alloying elements on the elastic properteis and magnetic behavior of $\mathrm{Ni3Al}$, Comput. Mater. Sci., 101(2015)39-46 
[8] A.V. Ruban, H.L. Skriver, Calculated site substitution in Y'-Ni3Al, Solid State Communications, 99 (1996) 813-817.

[9] C. Jiang, B. Gleeson, Site preference of transition metal elements in Ni3Al, Scripta Mater., 55 (2006) 433-436.

[10] C. Jiang, D.J. Sordelet, B. Gleeson, Site preference of ternary alloying elements in Ni3Al: A first-principles study, Acta Mater., 54 (2006) 1147-1154.

[11] C. Wagner, W. Schottky, Theorie der geordneted Mischpasen, Z. Physik. Chem. B, 11 (1930) 163.

[12] Z. Yuan, S. Song, R.G. Faulkner, Z. Yu, Combined effects of cerium and boron on the mechanical properteis and oxidation behaviour of Ni3Al alloys, J. Mater. Sci., 33 (1998) 463469.

[13] M.J. Donachie, Superalloys: A Technical Guide, Second ed., ASM International, 2002.

[14] Y. Zhexi, S. Shenhua, X. Tianhui, Z. Xiju, Y. Zongsen, Influence of cerium on ductility of Ni3Al, J. Mater. Sci. Lett., 13 (1994) 1717-1719.

[15] H.-E. Huang, C.-H. Koo, Effect of zirconium on microstructure and mechanical properteis of cast fine-grain CM 247 LC superalloy, Mater. Trans. 45 (2004) 554-561.

[16] P.J. Zhou, J.J. Yu, X.F. Sun, H.R. Guan, Z.Q. Hu, Roles of Zr and Y in cast microstructure of M951 nickel-based superalloy, Trans. Nonferr. Metal. Soc. China, 22 (2012) 1594-1598.

[17] D.M. Anliker, J.B. Newkirk, The effects of Cerium on the microstructure of INCO 901 superalloy, Metallurgical Transactions A, 7A (1976) 1711-1718.

[18] C. C. Fischer et al., Predicting crystal structure by merging data mining with quantum mechanics, Nature Mater 5 (2006) 641.

[19] G. H. Jóhannesson et al., Combined electronic structure and evolutionary search approach to materials design, Physical Review Letters 88 (2002) 255506.

[20] M. D'Avenzac, and A. Zunger, Identifying the minimum-energy atomic configuration on a lattice: Lamarckian twist on Darwinian evolution, Physical Review B 78 (2008) 064102.

[21] M. de Jong, et al, A statistical learning framework for materials science: application to elastic moduli of k-nary inorganic polycrystalline compounds, 6 (2016) 34256.

[22] M. de Jong, et al, Charting the complete elastic properties of inorganic crystalline compounds, Scientific Data, 2 (2015) 15009.

[23] J. R. Alvarez, and P. Rez, Calculation of electronic properties of boundaries in Ni3Al, Acta Materialia 49 (2001) 795.

[24] W. Zhou, H. Wu, and T. Yildirim, Electronic, dynamical and thermal properteis of ultraincompressible superhard rhenium diboride: a compined first-principles and neutro scattering study, Physical Review B 76, (2007) 184113.

[25] S. F. Matar, and M. A. Subramanian, Calculated electronic properties of the mixed perovskite oxides: CaCu3T4O12 ( $\mathrm{T}=\mathrm{Ti}, \mathrm{Cr}, \mathrm{Mn}, \mathrm{Ru})$ within the DFT, Materials Letters 58, (2004) 746.

[26] L. Cheng-Bin et al., First principles study on the charge density and the bulk modulus of the transition metals and their carbides and nitrides, Chinese Physics 14 (2005) 2287.

[27] P. Caron, O. Lavigne, Recent studies at Onera on superalloys for single crystal turbine blades, Aersosp. Lab, 3 (2011) 1-14.

[28] G. Kresse, J. Furthmüller, Efficient iterative schemes for ab initio total-energy calculations using a plane-wave basis set, Phys. Rev.B, 54 (1996) 11169.

[29] G. Kresse, J. Furthmüller, Efficiency of ab-initio total energy calculations for metals and semiconductors using a plane-wave basis set, Comput. Mater. Sci., 6 (1996) 15-50.

[30] G. Kresse, J. Hafner, Ab initio molecular-dynamics simulation of the liquid-metalamorphous-semiconductor transition in germanium, Phys. Rev. B, 49 (1994) 14251.

[31] G. Kresse, J. Hafner, Ab initio molecular dynamics for liquid metals, Phys. Rev. B, 47 (1993) 558. 
[32] J.P. Perdew, K. Burke, M. Ernzerhof, Generalized gradient approximation made simple, Phys. Rev. Lett., 77 (1996) 3865.

[33] J.P. Perdew, K. Burke, M. Ernzerhof, Generalized gradient approximation made simple, Phys. Rev. Lett., 78 (1997) 1396.

[34] P. E. Blöchl, Projector augmented-wave method, Phys. Rev. B ,50 (1994) 17953.

[35] H.J. Monkhorst, J.D. Pack, Special points for Brillouin-zone integration, Phys. Rev. B, 13 (1976) 5188-5192.

[36] S. R. Broderick, C. Suh, J. Provine, C. S. Roper, R. Maboudian, R. T. Howe, and K. Rajan, Application of principal component analsis to a full profile correlative analysis of FTIR spectra, Surf. Interface Anal. 44, (2012) 365-371.

[37] S. R. Broderick, J. R. Nowers, B. Narasimhan, and K. Rajan., Tracking chemical processing pathways in combinatorial polymer libraries via data mining, J. Comb. Chem. 12 (2010) 270277.

[38] M. Ashton, R.G. Hennig, S.R. Broderick, K. Rajan, S. Sinnott, Computational discovery of stable M(2)AX phases, Physical Review B, 94, (2016) 054116.

[39] A. Daffertshofer, C.J. Lamoth, O.G. Meijer, P.J. Beek, PCA in studying coordination and variability: a tutorial, Clinical Biomechanics, 19 (2004) 415-428.

[40] L. Ericksson, T. Byrne, E. Johansson, J. Trygg, C. Vikstrom, Multi- and Megavariate Data Analysis: Principles, Applications, Umetrics Ab, Umea, 2001.

[41] H. Berthiaux, V. Mosorov, L. Tomczak, C. Gatumel, J.F. Demeyre, Principal component analysis for characterising homogeneity in powder mixing using image processing techniques, Chemical Engineering and Processing, 45 (2006) 397-403.

[42] S. Wold, M. Sjostrom, and L. Eriksson, PLS-regression: a basic tool of chemometrics, Chemometrics and Intelligent Laboratory Systems 58 (2001) 109.

[43] D. V. Nguyen, D. M. Rocke, Tumor classification by partial least squares using microarray gene expression data, Bioinformatics, 18 (2002) 39-50.

[44] R. Rosipal, N. Kramer, Overview and recent advances in partial least squares, in Subspace, Latent Structure and Feature Selection Techniques, C. Saunders ed. Springer, Berlin/Heidelberg (2006) 34-51.

[45] P. Geladi, B. R. Kowalski, Partial least-squares regression: a tutorial, Analytica Chimica Acta, 185 (1986) 1-17.

[46] S. de Jong, SIMPS, an alternative approach to partial least squares regression, Chemometrics and Intelligent Laboratory Systems, 18 (1993) 251-263.

[47] A. Phatak, S. D. Jong, The geometry of partial-least squares, J. Chemometrics, 11 (1997) 311-338.

[48] P.V. Balachandran, S.R. Broderick, K. Rajan, Identifying the 'inorganic gene' for hightemperature piezoelectric perovskites through statistical learning, Proceedings Royal Society A, 467 (2011) 2271-2290.

[49] O. Wodo, S. Broderick, K. Rajan, Microstructural informatics for accelerating the discovery of processing-microstructure-property relationships, MRS Bulletin, 41 (2016) 603-609.

[50] S. Broderick, K. Rajan, Informatics derived materials databases for multifunctional properties, Science and Technology of Advanced Materials, 16 (2015) 013501.

[51] A. A. Oni, X. Sang, S.V. Raju, S. Dumpala, S. Broderick, A. Kumar, S. Sinnott, S. Saxena, K. Rajan, J.M. LeBeau, Large area strain analysis using scanning transmission electron microscopy across multiple images, App. Phys. Lett. 106 (2015) 011601. 